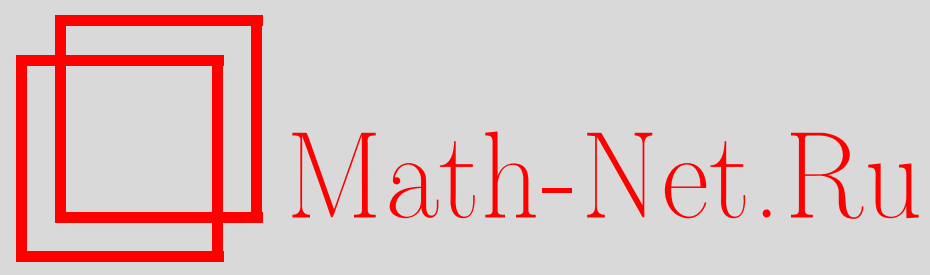

Н. А. Немировская, Теорема Фрухта для инверсных полугрупп, Матем. заметки, 1997, том 61, выпуск 2, 246-251

DOI: https://doi.org/10.4213/mzm1497

Использование Общероссийского математического портала Math-Net.Ru подразумевает, что вы прочитали и согласны с пользовательским соглашением http://www . mathnet.ru/rus/agreement

Параметры загрузки:

IP: 52.6 .47 .48

26 апреля 2023 г., 13:08:17

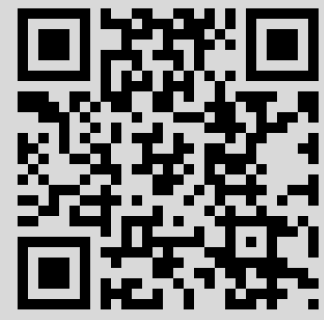




\title{
ТЕОРЕМА ФРУХТА ДЛЯ ИНВЕРСНЫХ ПОЛУГРУПП
}

\author{
Н. А. Немировская
}

\begin{abstract}
В заметке рассматривается задача о представлении конечных инверсных полугрупп частичными преобразованиями графов. Вводятся понятия взвешенного граффа и его весовых частичных автоморфизмов. Основньм результатом является теорема о том, что каждая конечная инверсная полугруппа изоморфна полугруппе весовых частичных автоморфизмов некоторого взвешенного граффа. Это утверждение является естественњым обобщением теоремы Фрухта для групп.

Библиограффия: 5 названий.
\end{abstract}

В 1936 г. Кёниг [1] поставил вопрос: когда данная абстрактная групша изоморфна группе автоморфизмов некоторого графа? Ответ был дан в 1938 г. Фрухтом [2]: каждая конечная группа представима как группа автоморфизмов некоторого графа.

C 60-х годов начинают рассматривать и другие алгебраические структуры, связанные с графами: полугрупшы эндоморфизмов графов, полугруппы частичных эндоморфизмов и др. (см. обзор [3]). Вопрос Кёнига имеет смысл и для этих структур. Один из последних результатов в этом направлении - полученные в [4] необходимые и достаточные условия того, что полугрупша частичных преобразований множества $X$ является полугруппой частичных эндоморфизмов некоторого графа с множеством вершин $X$.

Далее под графом понимается конечный неориентированньй граф без петель. Частичный автоморфизм графа Г - это взаимно однозначное (возможно, не везде определенное) отображение $\varphi: V(\Gamma) \rightarrow V(\Gamma)$ множества $V(\Gamma)$ вершин графа $\Gamma$, удовлетворяющее условию: для любых вершин $x$ и $y$ из области определения $\operatorname{dom} \varphi$ отображения $\varphi$ вершины $\varphi(x)$ и $\varphi(y)$ соединены ребром тогда и только тогда, когда ребром соединены $x$ и $y$. Относительно композиции множество PAut $\Gamma$ всех частичных автоморфизмов графа Г образует полугрупу, которая, как легко видеть, будет инверсной. Однако, далеко не каждая конечная инверсная полугруппа изоморфна PAut $Г$ для некоторого графа $Г$. Одна из причин - PAut Г содержит слишком много идеалов. В частности, PAut никогда не будет группой.

Граф Г назовем взвешенным, если задано сюръективное отображение $\omega: V(\Gamma) \rightarrow P$, где $P$ - некоторая нижняя полурешетка; $\omega(x)$ будем назьвать весом вериины $x \in V(\Gamma)$. Подмножество вершин $W \subseteq V(\Gamma)$ взвешенного графа $Г$ назовем конусом, если $W=$ $\{x \in V(\Gamma) \mid \omega(x) \leqslant \alpha\}$ для некоторого $\alpha \in P$. Частичный автоморфизм $\varphi \in \operatorname{PAut~} \Gamma$

Работа выполнена при частичной поддержке Международного научного фонда, грант GSU 041049. 
назовем весовым, если его область определения $\operatorname{dom} \varphi$ и область значений ran $\varphi-$ конусы и $\varphi$ сохраняет порядок весов вершин, т.е. для любых $a, b \in \operatorname{dom} \varphi$ из $\omega(a)=\omega(b)$ следует $\omega(\varphi(a))=\omega(\varphi(b))$ и из $\omega(a)<\omega(b)$ следует $\omega(\varphi(a))<\omega(\varphi(b))$.

ПРЕДЛОЖЕНИЕ. Совокупность РAut ${ }_{\omega} \Gamma$ всех весовых частичных автоморфизмов графа Г образует инверсную подполугруппу полугруппь PAut $\Gamma$.

ДокАЗАТЕЛЬСТво. Очевидно, что для любого $\varphi \in$ PAut $_{\omega} \Gamma$ инверсньй к нему элемент $\psi$ также принадлежит PAut $_{\omega} \Gamma$, и композищия $\varphi \psi$ элементов из PAut $_{\omega} \Gamma$ сохраняет порядок весов вершин. Осталось показать, что $\operatorname{dom} \varphi \psi$ и $\operatorname{ran} \varphi \psi-$ конусы. Пусть конусы $\operatorname{ran} \varphi$ и $\operatorname{dom} \psi$ определяются соответственно элементами $\alpha$ и $\beta$ из $P$. Поскольку $P-$ нижняя полурешетка, то из определения конуса следует, что

$$
\operatorname{ran} \varphi \cap \operatorname{dom} \psi=\{x \mid \omega(x) \leqslant \gamma\}
$$

где $\gamma=\inf (\alpha, \beta)$. Выберем из $\operatorname{ran} \varphi \cap \operatorname{dom} \psi$ произвольную вершину $c$ веса $\gamma$. Тогда

$$
\operatorname{dom} \varphi \psi=\{y \mid \omega(y) \leqslant \omega(\varphi(c))\}, \quad \operatorname{ran} \varphi \psi=\{z \mid \omega(z) \leqslant \omega(\varphi(c))\} .
$$

Предложение доказано.

Заметим, что если $|P|=1$, то PAut $\omega$ Г совпадает с Aut $\Gamma$.

Основным результатом заметки является

ТЕОремА. Для каждой конечной инверсной полугруппы $S$ существует такой взвешенный граф $\Gamma$, что $\operatorname{PAut}_{\omega} \Gamma \simeq S$.

Для доказательства нам понадобятся некоторые вспомогательные конструкции и леммы. Сначала сопоставим конечной инверсной полугрупе $S$ цветной орграф $\widetilde{\Gamma}$ с множеством вершин $S$, в котором вершины $a$ и $b$ соединены стрелкой из $a$ в $b$ тогда и только тогда, когда $b \in S a$, причем эта стрелка имеет цвет $b a^{-1}$. Рассмотрим представление Вагнера-Престона полугруппы $S$ частичными подстановками множества $S$ (см. [5, теорема 1.20]): элементу $a \in S$ сопоставляется частичная подстановка $\varphi_{a}$ с областью определения $\operatorname{dom} \varphi_{a}=S a^{-1}$, переводящая каждый элемент $x \in S a^{-1}$ в элемент $x a$. При этом область значений $\varphi_{a} \operatorname{ran} \varphi_{a}=S a$.

ЛЕмма 1. Для каждого $a \in S \quad \varphi_{а}$ является частичным автоморфизмом орграфа $\widetilde{\Gamma}$, сохраняюшим цвета ребер.

ДокАЗАТЕльство. а) Пусть $x, y \in S a^{-1}$ и из $x$ в $y$ идет стрелка цвета $y x^{-1}$. По определению орграфа $\widetilde{\Gamma}$ и отображения $\varphi_{a}$ существуют такие $s$ и $t$ из $S$, что $y=s a^{-1}$ и $y=t x$. Тогда $y a=(t x) a=t(x a) \in S(x a)$ и $(y a)(x a)^{-1}=y a a^{-1} x^{-1}=s a^{-1} a a^{-1} x^{-1}=$ $s a^{-1} x^{-1}=y x^{-1}$, т.е. из $x a$ в $y a$ тоже идет стрелка цвета $y x^{-1}$.

b) Пусть теперь $x, y \in S a^{-1}$ и $y \notin S x$. Предположим, что из $x a$ в уа идет стрелка. Поскольку $x a$ и уа принадлежат $\operatorname{dom} \varphi_{a^{-1}}$ и существуют такие $u, s \in S$, что $x=u a^{-1}$, $y=s a^{-1}$, то по доказанному вьше должна идти стрелка из $x=u a^{-1}=u a^{-1} a a^{-1}=$ $\varphi_{a^{-1}}(x a)$ в $y=s a^{-1}=s a^{-1} a a^{-1}=\varphi_{a^{-1}}(y a)$. А это противоречит условию $y \notin S x$.

Пусть ' $\leqslant$ ' - естественный частичньй порядок на множестве $I(S)$ идемпотентов полугруппы $S$. Из леммы 1.19 из $[5]$ следует, что $(I(S), \leqslant)$ будет нижней полурешеткой. Каждой вершине $a$ орграфа $\widetilde{\Gamma}$ припишем вес $\omega(a)=a^{-1} a$. Заметим, что $\omega(a)=\omega\left(a^{-1} a\right)$. 
ЛЕМма 2. Выполнено

a) $b \in S a \Longleftrightarrow \omega(b) \leqslant \omega(a)$;

b) $S b \subseteq S a \Longleftrightarrow \omega(b) \leqslant \omega(a)$;

c) $S b=S a \Longleftrightarrow \omega(b)=\omega(a)$.

ДокАЗАтЕльство. а) Поскольку $S a=S a^{-1} a$, то из $b=x a^{-1} a$ следует $b^{-1} b=$ $a^{-1} a x^{-1} x a^{-1} a$, откуда $\left(b^{-1} b\right)\left(a^{-1} a\right)=b^{-1} b$, т.е. $b^{-1} b \leqslant a^{-1} a$. Обратно, из $b^{-1} b \leqslant a^{-1} a$ следует $b^{-1} b=b^{-1} b a^{-1} a$, откуда $b=b a^{-1} a$, т.е. $b \in S a$.

b) следует из а), поскольку $b=b b^{-1} b \in S b$.

c) следует из b).

Лемма 3. $\varphi_{a^{-1} \text { a }}$ совпадает с тождественным отображением множества Sа на себя.

Доказательство следует из равенств $S \cdot\left(a^{-1} a\right)^{-1}=S a$ и $x a \cdot a^{-1} a=x a$.

В следующих двух леммах предполагается, что частичный автоморфизм $\varphi$ орграфа $\widetilde{\Gamma}$ сохраняет цвета ребер, а его область определения имеет вид $\operatorname{dom} \varphi=\{x \mid \omega(x) \leqslant \omega(a)\}$ для некоторого $a \in S$. Заметим, что при этих предположениях $a^{-1} a \in \operatorname{dom} \varphi$.

Лемма 4. Если $\varphi\left(a^{-1} a\right)-$ идемпотент полугруппы $S$, mо $\varphi=\varphi_{a^{-1} a}$.

ДокАЗАтЕльство. Из леммы 2 следует, что $\operatorname{dom} \varphi=S a$. Поскольку $S a=S a^{-1} a$, то для любого $y a \in S a$ из вершины $a^{-1} a$ в вершину уа идет стрелка цвета $y a$. Но тогда из $\varphi\left(a^{-1} a\right)$ в $\varphi(y a)$ также идет стрелка цвета $y a$, кроме того, $\varphi(y a)=t \cdot \varphi\left(a^{-1} a\right)$ для некоторого $t \in S$. С другой стороны, поскольку $\varphi\left(a^{-1} a\right)$ - идемпотент, то стрелка из $\varphi\left(a^{-1} a\right)$ в $\varphi(y a)$ имеет цвет

$$
\varphi(y a) \cdot\left(\varphi\left(a^{-1} a\right)\right)^{-1}=t \cdot \varphi\left(a^{-1} a\right) \cdot\left(\varphi\left(a^{-1} a\right)\right)^{-1}=t \cdot \varphi\left(a^{-1} a\right)=\varphi(y a) .
$$

Следовательно, для всех $y a \in S a \varphi(y a)=y a$, т.е. $\varphi$ действует на $S a$ тождественно и по лемме 3 совпадает с $\varphi_{a^{-1} a}$.

Лемма 5. Существует такой әлемент $b \in S$, что $\varphi=\varphi_{b}$.

ДокАЗАТЕЛЬСтво. Предположим, что такого элемента не существует. Тогда по лемме 4 элемент $c=\varphi\left(a^{-1} a\right)$ не является идемпотентом. Заметим, что $\varphi(S a) \subseteq S c$. Рассмотрим частичный автоморфизм $\psi=\varphi \varphi_{c-1}$. Если бы для некоторого $y \in S$ выполнялось равенство $\psi=\varphi_{y}$, то, вопреки предположению, имели бы

$$
\varphi=\varphi \cdot \varphi_{c^{-1} c}=\varphi \cdot \varphi_{c^{-1}} \cdot \varphi_{c}=\psi \varphi_{c}=\varphi_{y} \varphi_{c}=\varphi_{y c}
$$

Поскольку $\psi$ сохраняет цвета ребер и $\operatorname{dom} \psi=\operatorname{dom} \varphi$, то по лемме $4 \psi\left(a^{-1} a\right)$ не является идемпотентом. Но

$$
\psi\left(a^{-1} a\right)=\left(\varphi \varphi_{c^{-1}}\right)\left(a^{-1} a\right)=\varphi_{c^{-1}}(c)=c c^{-1} .
$$

Полученное противоречие доказьвает лемму.

ЛЕмма 6. Каждый частичный автоморфизм ч орәрафа $\widetilde{\Gamma}$ сохраняет порядок весов вериин. 
ДокАЗАтЕЛЬство. Пусть $a, b \in \operatorname{dom} \varphi$ и $\omega(a)=\omega(b)$, т.е. по лемме $2 a \in S b$ и $b \in S a$. Тогда по построению орграфа $\widetilde{\Gamma} a$ и $b$ соединены стрелкой из $a$ в $b$ и стрелкой из $b$ в $a$. Отсюда $\varphi(a)$ и $\varphi(b)$ соединены стрелками из $\varphi(a)$ в $\varphi(b)$ и из $\varphi(b)$ в $\varphi(a)$. Из леммы 2 и построения орграфа $\widetilde{\Gamma}$ следует, что $\omega(\varphi(a))=\omega(\varphi(b))$.

Аналогично, для любых $a, b \in \operatorname{dom} \varphi$ из $\omega(a)<\omega(b)$ следует $\omega(\varphi(a))<\omega(\varphi(b))$.

Из лемм 1,2 и 6 следует, что для любого $a \in S$ частичный автоморфизм $\varphi_{a}$ орграфа $\widetilde{\Gamma}$ сохраняет цвета́ ребер и является весовым. Отсюда и из леммы 5 получаем, что сохраняющие цвета́ ребер весовые частичные автоморфизмы орграфа $\widetilde{\Gamma}$ образуют полугруппу, изоморфную $S$.

ДокАЗАТЕЛЬСТво тЕоРЕмЫ. Пусть $\Delta-$ множество цветов ребер орграфа $\widetilde{\Gamma}$. Зададим инъективные отображения

$$
\Delta \rightarrow\{n \in \mathbb{N} \mid n \geqslant 2\}, \quad x \mapsto n_{x}, \quad \text { и } \quad I(S) \rightarrow\{m \in \mathbb{N} \mid m>M\}, \quad e \mapsto m,
$$

где

$$
M=\max _{x \in \Delta} n_{x}
$$

Теперь следуюшим образом преобразуем орграф $\widetilde{\Gamma}$ во взвешенньй граф Г. Из того, что $a=a a^{-1} a \in S a$, следует, что орграф $\widetilde{\Gamma}$ содержит в вершине $a$ петлю цвета $a a^{-1}$. Если $a \neq 0$, то заменяем эту петлю подграфом $\Gamma_{a}$, приведенным на рис. 1 , в противном случае $(a=0)$ считаем $a$ вершиной без петли. Каждую стрелку из $a$ в $b(a \neq b)$ заменим подграфом $\Gamma_{a, b}$, приведенньм на рис. 2 . Всем новым вершинам этих подграфов приписьваем $\operatorname{Bec} \omega(a)=a a^{-1}$.

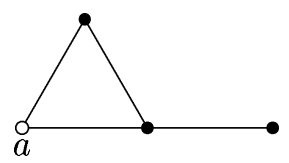

Рис. 1

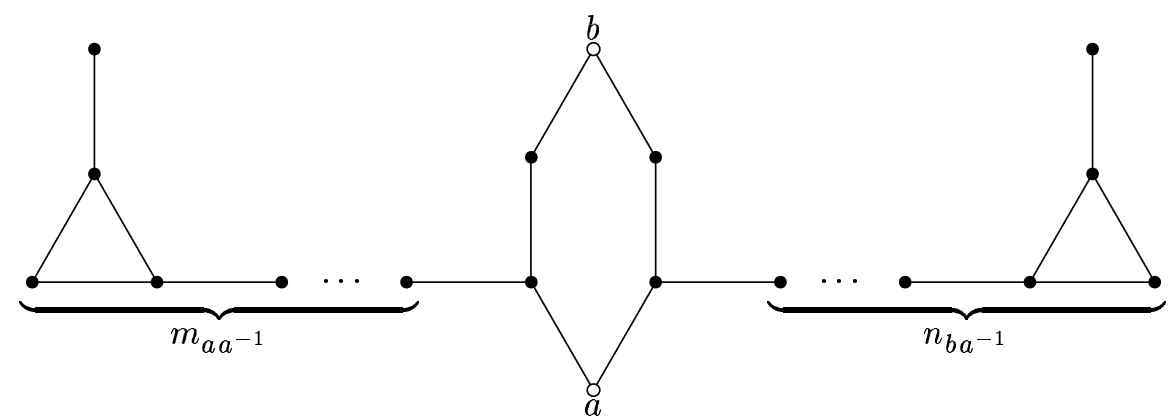

Рис. 2

Заметим, что для любого элемента $b \in S a^{-1}$ из $b a=0$ следует $b=0$. Отсюда и из построения графа $\Gamma$ следует, что для любого $0 \neq b \in S a^{-1}$ существует единственный изоморфизм $\psi_{b}: \Gamma_{b} \rightarrow \Gamma_{\varphi_{a}(b)}$, удовлетворяюший условию $\psi_{b}(b)=\varphi_{a}(b)$. Кроме того, 
для любых $b \in S a^{-1}$ и $c \in S b$ из построения граффа $\Gamma$ следует существование единственного изоморфизма $\psi_{b, c}: \Gamma_{b, c} \rightarrow \Gamma_{\varphi_{a}(b), \varphi_{a}(c)}$. Теперь для каждого $a \in S$ следующим образом определим частичное отображение $\varphi_{a}^{*}$ вершин графа $\Gamma$ :

$\varphi_{a}^{*}(x)= \begin{cases}\varphi_{a}(x), & \text { если } x \in S a^{-1} ; \\ \psi_{b}(x), & \text { если для некоторого } b \in S a^{-1} x \text { является вершиной графа } \Gamma_{b} ; \\ \psi_{b, c}(x), & \text { если для некоторых } b, c \in S a^{-1} x \text { является вершиной графа } \Gamma_{b, c} ; \\ & \text { не определено в остальных случаях. }\end{cases}$

Поскольку для любого $b \in S a^{-1} \varphi_{a}(b)=\psi_{b}(b)=\psi_{b, c}(b)$, то отображение $\varphi_{a}^{*}$ определено корректно.

Легко проверяется, что $\varphi_{a}^{*}$ является частичным автоморфизмом графа $Г$ и сохраняет порядок весов. Докажем, что

$$
\operatorname{dom} \varphi_{a}^{*}=\left\{x \in V(\Gamma) \mid \omega(x) \leqslant \omega\left(a^{-1}\right)\right\}
$$

Действительно, вес $\omega(x)$ любой вершины $x \in \operatorname{dom} \varphi_{a}^{*}$ совпадает с весом одного из элементов $b \in S a^{-1}$. Но тогда по лемме $2 \omega(x) \leqslant \omega\left(a^{-1}\right)$. Обратно, если $\omega(x) \leqslant \omega\left(a^{-1}\right)$, то по построению существует такой $b \in S a^{-1}$, что $\omega(x)=\omega(b)$ и $x$ является вершиной либо графа $\Gamma_{b}$, либо одного из графов $\Gamma_{b, c}$. Поскольку $c \in S b$, то из $b \in S a^{-1}$ следует $c \in S a^{-1}$. Следовательно, в обоих случаях $x \in \operatorname{dom} \varphi_{a}^{*}$.

Из $\operatorname{ran} \varphi_{a}^{*}=\operatorname{dom} \varphi_{a^{-1}}^{*}$ следует, что $\operatorname{ran} \varphi_{a}^{*}$ также является конусом. Поэтому $\varphi_{a}^{*} \in \operatorname{PAut}_{\omega} \Gamma$.

Очевидно, что отображение $a \mapsto \varphi_{a}^{*}$ является гомоморфизмом $S$ в полугруппу PAut $_{\omega} \Gamma$. Поскольку на вершинах из $\widetilde{\Gamma} \varphi_{a}^{*}$ совпадает с $\varphi_{a}$, то этот гомоморфизм будет инъективньп. Для завершения доказательства теоремы осталось показать, что каждьй весовой частичньй автоморфизм графа $Г$ имеет вид $\varphi_{a}^{*}$.

Пусть $\varphi \in \operatorname{PAut}_{\omega} \Gamma$. Тогда

$$
\operatorname{dom} \varphi=\{x \in V(\Gamma) \mid \omega(x) \leqslant \omega(e)\}
$$

где $e$ - некоторьй идемпотент из $S$. Если $e=0$, то $\operatorname{dom} \varphi=\{0\}$. Так как по построению графа $\Gamma\{0\}$ - единственньй одноэлементный конус, то $\varphi(0)=0$, т.е. $\varphi=\varphi_{0}^{*}$.

Вообще, если $S$ - полугруппа с нулем, то для любого весового частичного автоморфизма $\varphi \in \operatorname{PAut}_{\omega} \Gamma 0 \in \operatorname{dom} \varphi$ и $\varphi(0)=0$.

Пусть теперь $e \neq 0$. Поскольку для любого $b, 0 \neq b \in S e$, все вершины подграфов $\Gamma_{b}$ и $\Gamma_{b, c}$ имеют вес $\leqslant \omega(b)$, то частичньй автоморфизм $\varphi$ определен на всех вершинах таких подграфов. Заметим, что для любого $b \neq 0|S b|>1$, т.е. орграф $\widetilde{\Gamma}$ содержит петлю в вершине $b$ и стрелку из $b$ в $c, b \neq c \in S b$. Поэтому любая вершина $b, 0 \neq b \in S e$, в порожденном множеством $\operatorname{dom} \varphi$ подграфе графа $\Gamma$ имеет степень $\geqslant 4$, в то время как любая вершина из $V(\Gamma) \backslash V(\widetilde{\Gamma})$ имеет степень не больше 3. Отсюда и из сделанного выше замечания следует, что $\varphi$ переводит вершины орграфа $\widetilde{\Gamma}$ в вершины орграфа $\widetilde{\Gamma}$, а вершины из $V(\Gamma) \backslash V(\widetilde{\Gamma})$ - в вершины из $V(\Gamma) \backslash V(\widetilde{\Gamma})$. В частности, можно говорить о сужении $\widetilde{\varphi}$ частичного автоморфизма $\varphi$ на орграф $\widetilde{\Gamma}$.

Очевидно, что $\widetilde{\varphi}$ сохраняет порядок весов вершин и $\operatorname{dom} \widetilde{\varphi}$ и $\operatorname{ran} \widetilde{\varphi}-$ конусы. Кроме того, если вершины $a, b \in \operatorname{dom} \widetilde{\varphi}$ соединены стрелкой из $a$ в $b$, то $\varphi$ должен переводить 
граф $\Gamma_{a, b}$ в подграф графа $\Gamma_{\varphi(a), \varphi(b)}$. Но граф $\Gamma_{a, b}$ изоморфен некоторому подграфу графа $\Gamma_{c, d}$ тогда и только тогда, когда $\Gamma_{a, b} \simeq \Gamma_{c, d}$. Отсюда, в частности, следует, что стрелки из $a$ в $b$ и из $\widetilde{\varphi}(a)$ в $\widetilde{\varphi}(b)$ одного цвета. Поэтому $\widetilde{\varphi}$ является сохраняющим цвета́ ребер весовым частичным автоморфизмом орграфа $\widetilde{\Gamma}$, т.е. $\widetilde{\varphi}=\varphi_{a}$ для некоторого $a \in S$. Поскольку $\widetilde{\varphi}_{a}^{*}=\varphi_{a}$ и из $\widetilde{\varphi}_{1}=\widetilde{\varphi}_{2}$ следует $\varphi_{1}=\varphi_{2}$, то $\varphi=\varphi_{a}^{*}$.

Киевский государственный университет

Поступило

им. Т. Шевченко

\section{СПИСОК ЦИТИРОВАННОЙ ЛИТЕРАТУРЫ}

[1] König D. Theorie der endlichen und unendlichen Graphen. Leipzig, 1936.

[2] Frucht R. Herstellung Von Graphen mit Vorgegebener abstrakten Gruppe // Compositio Math. 1938. V. 6. P. 239-250.

[3] Molchanov V.A. Semigroups of mapping on graphs // Semigroup Forum. 1983. V. 27. P. 155-199.

[4] Molčanov V. A. Concrete characterization of partial endomorphism semigroups of graphs // Acta Sci. Math. 1987. V. 51. P. 349-363.

[5] Клиффорд Г., Престон А. Алгебраическая теория полугрупп. Т. 1. М.: Мир, 1972. 